\title{
Posterior minimally invasive scoliosis surgery versus the standard posterior approach for the management of adolescent idiopathic scoliosis: an updated meta-analysis
}

\author{
Honghao Yang ${ }^{\dagger},{\text { Xiangyuan } \mathrm{Jia}^{\dagger} \text { and Yong Hai }}^{*}$ (1)
}

\begin{abstract}
Background: Surgical management of adolescent idiopathic scoliosis (AIS) can be performed using standard posterior spinal fusion (PSF) or with a posterior minimally invasive approach. Minimally invasive scoliosis surgery (MISS) has several theoretical advantages, such as less tissue dissection, less blood loss, and earlier recovery. However, the difference in safety and effectiveness between MISS and PSF still needs to be clarified. This updated meta-analysis aimed to compare the outcomes of MISS and standard PSF for the management of AIS.
\end{abstract}

Methods: A comprehensive literature search of PubMed, EMBASE, MEDLINE, and Cochrane Library without time restriction was performed to identify relevant studies. MISS and PSF were compared in terms of radiographic parameters, estimated blood loss (EBL), blood transfusion rate, operative time (ORT), length of hospital stay (LOS), overall Scoliosis Research Society-22 (SRS-22) score, postoperative pain, and complication rate.

Results: A total of seven studies comprising 767 patients (329 MISS and 438 PSF) with AIS were included. MISS and PSF yielded comparable deformity correction at the last follow-up. There were no significant differences in the overall SRS-22 scores or complication rates between the groups. Nevertheless, greater restoration of thoracic kyphosis (WMD, 2.98; $95 \% \mathrm{Cl} 0.58$ to 5.37, $P=0.015)$, less $\mathrm{EBL}(\mathrm{WMD},-218.76 ; 95 \% \mathrm{Cl}-256.41$ to $-181.11, P<0.001)$, a lower blood transfusion rate $(\mathrm{RR}, 0.31 ; 95 \% \mathrm{Cl} 0.20$ to $0.48, P<0.001)$, a shorter $\operatorname{LOS}(\mathrm{WMD},-1.48 ; 95 \% \mathrm{Cl}-2.48$ to $-0.48, P=0.004)$, less postoperative pain (WMD, $0.57 ; 95 \% \mathrm{Cl} 0.16$ to $0.98, P=0.006$ ), and a longer ORT (WMD, 84.85; 95\% Cl 33.30 to $136.40, P=0.001$ ) were observed in the MISS group.

Conclusion: Despite its inherent technical challenges, MISS is a feasible and effective alternative to standard PSF for AIS patients with moderate and flexible curves. MISS was associated with adequate deformity correction, better restoration of sagittal alignment, less EBL, fewer transfusions, shorter LOS, and better pain management compared to PSF. Further research is required to determine the detailed indications for the MISS procedure.

Keywords: Minimally invasive scoliosis surgery, Posterior spinal fusion, Adolescent idiopathic scoliosis, Spinal deformity, Surgical management

*Correspondence: yong.hai@ccmu.edu.cn

†Honghao Yang and Xiangyuan Jia contribute equally to this study Department of Orthopedic Surgery, Beijing Chao-Yang Hospital, Capital Medical University, Gongti South Rd, No. 8, Beijing 100020, China

\section{Background}

Adolescent idiopathic scoliosis (AIS) is the most common spinal disorder affecting patients aged 10 years to maturity, and it has a high prevalence of $2 \%$ to $3 \%$ worldwide [1]. Management options include braces and original author(s) and the source, provide a link to the Creative Commons licence, and indicate if changes were made. The images or other third party material in this article are included in the article's Creative Commons licence, unless indicated otherwise in a credit line to the material. If material is not included in the article's Creative Commons licence and your intended use is not permitted by statutory regulation or exceeds the permitted use, you will need to obtain permission directly from the copyright holder. To view a copy of this licence, visit http://creativecommons.org/licenses/by/4.0/. The Creative Commons Public Domain Dedication waiver (http://creativeco mmons.org/publicdomain/zero/1.0/) applies to the data made available in this article, unless otherwise stated in a credit line to the data. 
specific exercises for mild scoliosis, while surgical intervention is needed for rapidly progressive cases [2]. The primary goals of spinal surgery are correcting deformity, restoring balance, and ultimately achieving solid arthrodesis [3]. Since the development of instrumentation, derotation maneuvers, and fusion techniques, open instrumented posterior spinal fusion (PSF) has been considered the standard procedure for the surgical treatment of AIS [4]. However, as a major surgery, PSF is associated with extensive blood loss, prolonged hospital stays, postoperative pain, and a risk of infection [5]. The long scar resulting from the incision is also a cosmetic problem for adolescent patients and may be accompanied by psychosocial distress [6]. Additionally, there are concerns regarding the paraspinal muscle morbidity caused by denervation [7]. Consequently, efforts have been made to limit the extent of surgical trauma.

Minimally invasive surgery (MIS) techniques have gained increasing popularity for the treatment of various traumatic or degenerative spinal disorders in the adult patient population $[8,9]$. MIS has also been shown to be comparable to PSF for correcting adult spinal deformity and has the additional benefit of reduced tissue damage, less bleeding, and earlier mobilization and discharge [10]. Previous feasibility studies of MIS for deformity correction and their reported advantages have convinced surgeons to attempt to use posterior minimally invasive techniques for AIS [11-13]. Although Sarwahi et al. and de Bodman et al. successfully performed three-incision minimally invasive scoliosis surgery (MISS) for moderate AIS patients, inherent technical challenges are present in this procedure [11, 14]. AIS patients have much larger curves than patients with degenerative scoliosis, and more levels require instrumentation. The use of multiple stab incisions of the skin limits the access needed to perform adequate facetectomies and fusion [15]. Additionally, due to the significant vertebral rotation in AIS, it is difficult to identify the ideal pedicle screw trajectory without a sufficient view of anatomical landmarks. The resulting need for repeated fluoroscopy results in greater radiation exposure for surgeons and patients, which is especially harmful to children [16]. Moreover, the presence of a rigid curve, vertebral rotation, limited visualization, and soft tissue may make contoured rod passage and reduction maneuvers challenging [17].

A meta-analysis comparing the outcomes of MISS and standard PSF in the management of moderate AIS was conducted by Alhammoud et al. in 2019, and they concluded that there were no differences in curve correction, postoperative pain, or length of hospital stay (LOS) between the two procedures [18]. However, their conclusions may be limited due to the small number of subjects (only four studies with 42 patients in the MISS group and 65 patients in the PSF group were included). Since new comparative studies with more subjects have been published since that meta-analysis, we considered that an updated meta-analysis was necessary to provide valuable guidance regarding current surgical options for AIS [19-21].

This updated meta-analysis aimed to compare the radiographic outcomes, surgical information, clinical outcomes, and complications of MISS and standard PSF for the management of AIS.

\section{Methods}

This study was designed according to the Preferred Reporting Items for Systematic Reviews and Meta-Analyses (PRISMA) guidelines [22]. This study is registered with PROSPERO (ID: CRD42020217823).

\section{Search strategy}

The PubMed, EMBASE, MEDLINE, and Cochrane Library databases were searched using the following terms: (minimally invasive) AND (adolescent idiopathic scoliosis).

The literature search was last updated on April 26, 2021. Two reviewers (H.Y. and X.J.) independently screened titles, abstracts, and full texts. Any differences that arose were settled by discussion with a third party (Y.H.).

\section{Inclusion and exclusion criteria}

The inclusion criteria in this meta-analysis were as follows: (1) target population: patients diagnosed with AIS; (2) intervention: MISS procedures were performed; (3) comparison: patients undergoing standard PSF procedures; and (4) outcomes: radiographic outcomes at the last follow-up, surgical information, clinical outcomes, and complications.

The exclusion criteria were as follows: (1) patients with a nonidiopathic etiology (e.g., neuromuscular or congenital scoliosis) and those undergoing revision spinal surgery; (2) patients undergoing thoracoscopic or laparoscopic surgery; (3) noncomparative studies; (4) reviews, case reports, and biomechanical studies; (5) duplicated publications; and (6) articles that were not published in English.

\section{Assessment of study quality}

The quality of the studies was assessed independently by two reviewers (H.Y. and X.J.) using the NewcastleOttawa Scale (NOS), as recommended for retrospective studies by Cochrane Handbooks version 5.2.0 [23]. 


\section{Outcomes}

Radiographic outcomes were the main curve Cobb angle and its correction rate, thoracic kyphosis (TK), lumbar lordosis (LL), coronal balance (CB), and sagittal vertical axis (SVA) at the last follow-up. Surgical information included estimated blood loss (EBL), blood transfusion rate, and operative time (ORT). The clinical outcomes were LOS, overall Scoliosis Research Society-22 (SRS22) score, SRS-22 self-image/appearance score, and postoperative pain based on the SRS-22 pain score or the visual analog scale (VAS) score. Complications included the overall complication rate, surgical site infection, and hardware failure.

\section{Data extraction}

Data extraction was performed by two reviewers independently (H.Y. and X.J.). Demographic and clinical information, including age, sex, curve types according to Lenke classification, and the number of fusion levels, was extracted; additionally, the sample size of each study was recorded [24]. This meta-analysis was performed for 17 variables. Continuous outcomes included the main curve Cobb angle and its correction rate, TK, LL, CB, SVA, EBL, ORT, LOS, overall SRS-22 score, SRS-22 self-image/ appearance score, SRS-22 pain score, and VAS score. Because a greater SRS-22 pain score indicates less pain, while a greater VAS score indicates more severe pain, we used the negative value of VAS scores in the data analysis of postoperative pain [25, 26]. Dichotomous outcomes included the blood transfusion rate, overall complication rate, surgical site infection rate, and hardware failure rate. Outcomes reported by at least three studies were analyzed.

\section{Data analysis}

All statistical analyses were performed using Stata 15.1. For continuous outcomes, the weighted mean difference (WMD) was utilized to estimate the effect. The effect measure of dichotomous outcomes was displayed as risk ratio (RR). Statistical heterogeneity among studies was evaluated using the I-square test and Cochran's Q test. If the $\mathrm{I}^{2}$ value was less than $50 \%$ and the $P$-value was greater than 0.1 , a fixed-effects model was performed. If the $\mathrm{I}^{2}$ value was greater than $50 \%$ or the $P$-value was less than 0.1 , a sensitivity analysis was applied to assess the impact of each study. A Galbraith plot was used to visualize the heterogeneity [27]. If the $\mathrm{I}^{2}$ and the $P$-value could be reduced to less than $50 \%$ and 0.1 , respectively, following the exclusion of one or two studies, these studies were omitted, and a fixed-effects model was used. Influence analysis was performed to investigate whether the exclusion of these studies would cause the effect size to be overestimated or underestimated [28]. If the heterogeneity could not be significantly diminished using these methods, the random-effects model was used. Subgroup analysis was performed to explore the potential sources of heterogeneity.

\section{Assessment of publication bias}

Potential publication bias was assessed by the application of Egger's test at the $P<0.10$ level of significance [29]. If publication bias was indicated, we evaluated the number of missing studies in this meta-analysis using the trim-and-fill method and recalculated the pooled effect estimate with the addition of the missing hypothetical studies [30].

\section{Results \\ Study selection}

The systematic search yielded 92 studies, of which 41 were duplicates. Thirty-eight studies were excluded after screening of the title and abstract, and six studies were reasonably considered inappropriate after full-text review. Eventually, seven studies were finally included in this meta-analysis (Fig. 1) [15, 19-21, 31-33].

\section{Assessment of study quality}

The quality of the included studies was assessed according to the NOS (Table 1). Of the seven studies, three were of high quality, with a score of 8 , and four were of moderate quality, with a score of 7 .

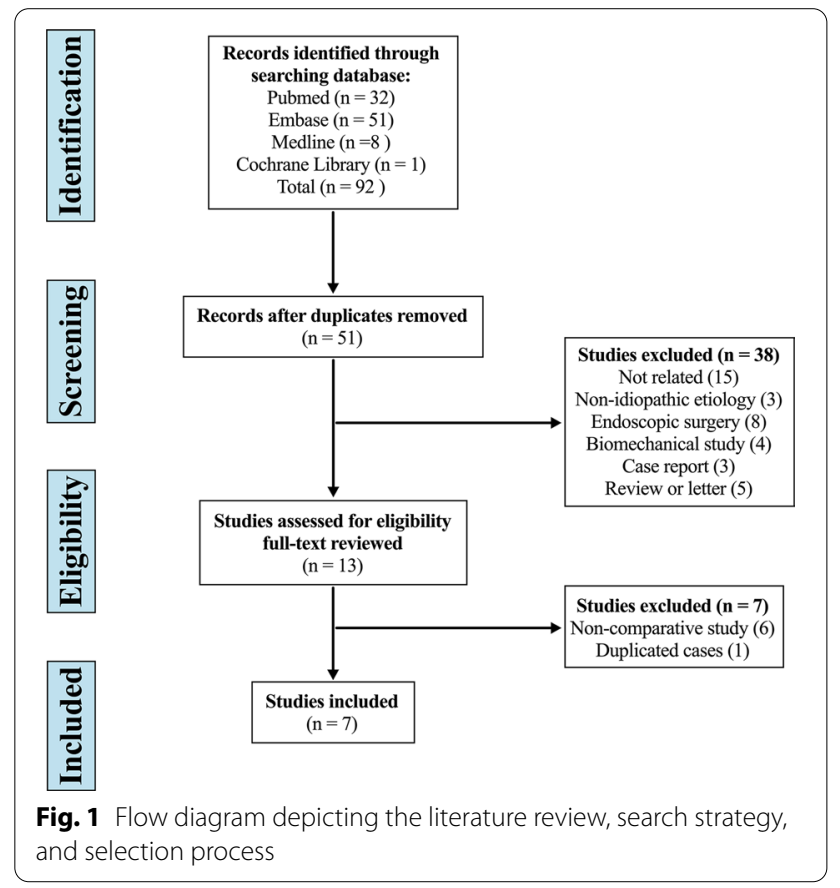


Table 1 Quality assessment of studies according to NewcastleOttawa Scale (NOS)

\begin{tabular}{llllll}
\hline Author & Year & Selection & Comparability & Exposure & Total Score \\
\hline Miyanji & 2015 & 3 & 2 & 2 & 7 \\
Sarwahi & 2016 & 3 & 2 & 2 & 7 \\
Zhu & 2017 & 3 & 2 & 3 & 8 \\
Urbanski & 2019 & 3 & 2 & 2 & 7 \\
Si & 2020 & 3 & 2 & 3 & 8 \\
Yang & 2021 & 3 & 2 & 2 & 7 \\
Sarwahi & 2021 & 3 & 2 & 3 & 8 \\
\hline
\end{tabular}

\section{Characteristics of the included studies}

A total of seven studies comprising 767 patients with AIS were included. Of the patients, 329 underwent MISS procedures, and 438 underwent PSF procedures. The characteristics of the included studies are shown in Table 2 . There were no significant differences in demographic data, number of fusion levels, main curve Cobb angle, TK, LL, CB, or SVA at baseline between the two groups.

\section{Surgical technique}

As reported in the included studies, PSF was performed using a standard posterior approach with subperiosteal muscle dissection, facetectomy/decortication, pedicle screw insertion, rod placement, derotation, and correction maneuvers. MISS was performed using one to three small $(3-5 \mathrm{~cm})$ midline skin incisions. Facet joints were exposed using the Wiltse approach. Three to four segments (6-8 pedicle screws) were instrumented per skin incision, followed by subfascial rod placement and correction maneuvers with posterolateral fusion around the facets.

\section{Radiographic outcomes \\ Main curve Cobb angle}

Seven studies reported the main curve Cobb angle at the last follow-up, and significant heterogeneity was detected $\left(P<0.001, I^{2}=89.7 \%\right)$. However, further sensitivity analysis did not identify a study that could significantly affect the heterogeneity. Therefore, the random-effects model was applied. The pooled results revealed no significant difference between groups in the main curve Cobb angle at the last follow-up (WMD, 2.60; 95\% CI -0.01 to 5.20, $P=0.051)$ (Fig. 2A).

\section{Correction rate of main curve Cobb angle}

Seven studies reported the correction rate of the main curve Cobb angle, and significant heterogeneity was detected $\left(P<0.001, I^{2}=92.8 \%\right)$. Therefore, a further sensitivity analysis was performed. The Galbraith plot indicated that the source of heterogeneity was the studies by Miyaji et al. [31] and by Urbanski et al. [33] (Additional file 1). Miyaji et al. [31] emphasized that the correction rate of MISS in their study may have been impacted by the learning curve when applying this new technique, while the difference in the preoperative main curve Cobb angle between the MISS and PSF groups was $10.3^{\circ}$ in the study by Urbanski et al. [33], which was significantly greater than that of other studies (range $1.3^{\circ}$ $2.7^{\circ}$ ) and may have skewed the correction rate. When the studies by Miyanji et al. [31] and by Urbanski et al. [33] were omitted from the meta-analysis, the heterogeneity was not significant $\left(P=0.164, I^{2}=38.7 \%\right)$. An influence analysis indicated that the exclusion of the study by Miyaji et al. [31] would significantly affect the effect size (Additional file 2).

The remaining five studies included 302 patients in the MISS group and 411 patients in the PSF group. The pooled results revealed no significant difference in the correction rate between groups (WMD, $-0.01 ; 95 \% \mathrm{CI}$ -0.03 to $0.01, P=0.518)$.

\section{Thoracic kyphosis}

Seven studies reported TK at the last follow-up, and significant heterogeneity was detected $\left(P<0.001, I^{2}=79.7 \%\right)$. However, further sensitivity analysis did not identify a study that could significantly affect the heterogeneity. Therefore, the random-effects model was applied. The mean TK at the last follow-up was $25.80^{\circ}$ (95\% CI 21.82 to 29.77 ) in the MISS group and $22.71^{\circ}$ (95\% CI 20.77 to 24.66) in the PSF group. The pooled results revealed that the TK at the last follow-up was significantly greater in the MISS group than in the PSF group (WMD, 2.98; 95\% CI 0.58 to $5.37, P=0.015$ ) (Fig. $2 \mathrm{~B}$ ).

Lumbar lordosis, coronal balance, and sagittal vertical axis The pooled results revealed that there were no significant differences between groups in LL (WMD, -2.18; 95\% CI -6.08 to $1.71, P=0.272$ ), CB (WMD, -0.56 ; $95 \% \mathrm{CI}-1.41$ to $0.30, P=0.201$ ), or SVA (WMD, $1.13 ; 95 \% \mathrm{CI}-0.54$ to $2.80, P=0.186)$ at the last follow-up.

\section{Surgical information \\ Estimated blood loss}

Seven studies reported EBL, and significant heterogeneity was detected $\left(P=0.003, I^{2}=69.5 \%\right)$. Therefore, further sensitivity analysis was performed. The Galbraith plot indicated that the source of heterogeneity was the study by Yang et al. [20] (Additional file 3). The difference in EBL between the MISS and PSF groups was $1224.0 \mathrm{~mL}$ in the study by Yang et al. [20], which was significantly greater than that in other studies (range 166.7-333.3 mL). Additionally, Yang et al. [20] reported 


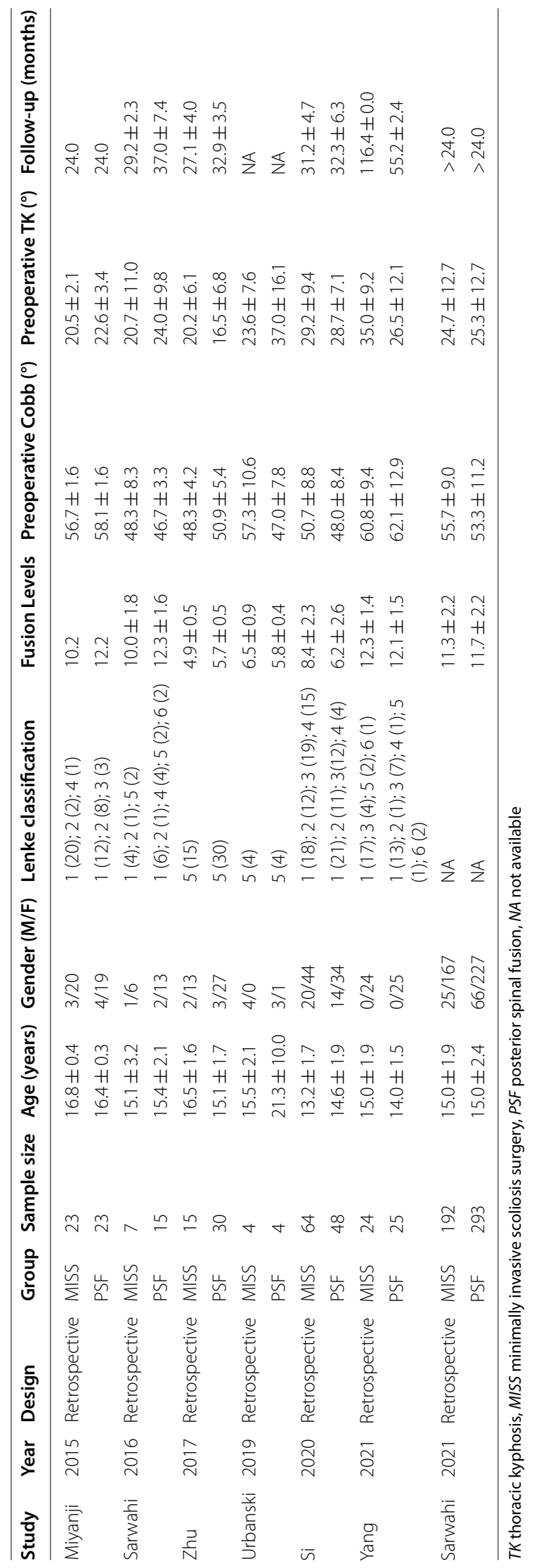




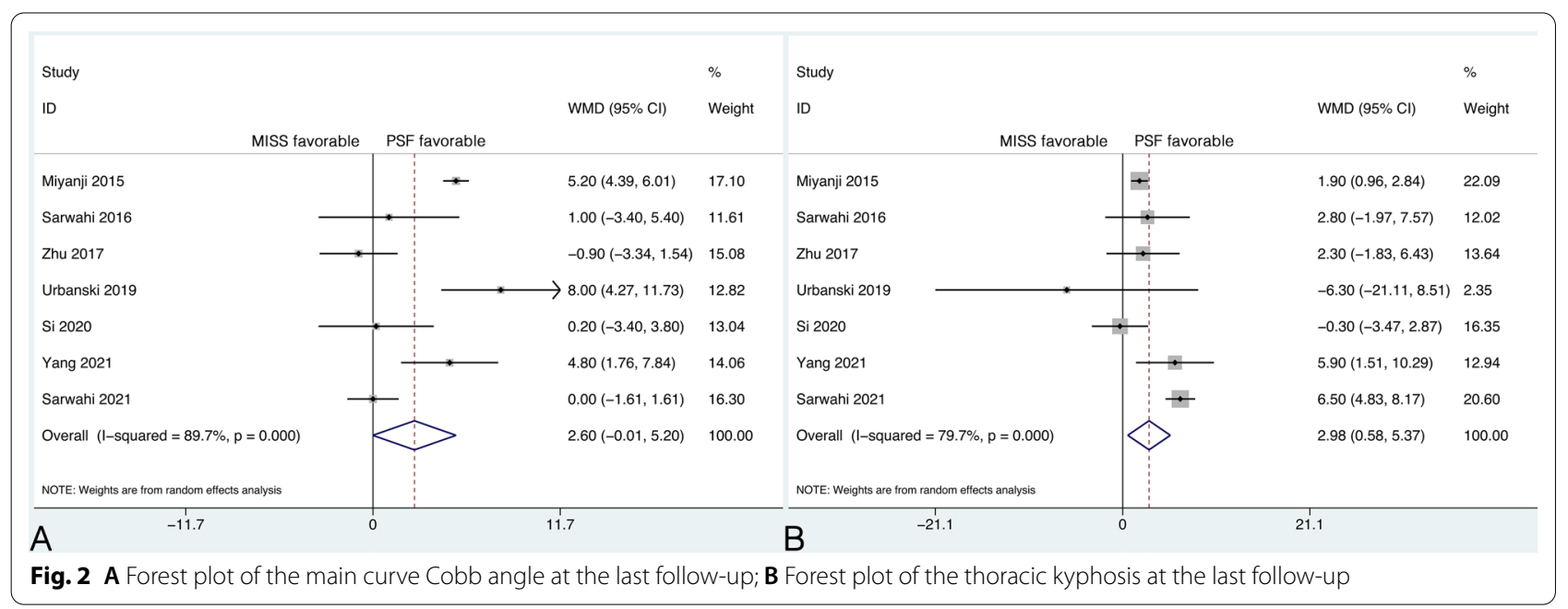

that the mean EBL in the PSF group was $2503.0 \mathrm{~mL}$, which was significantly higher than that reported in the other included studies (range 418.0-883.3 $\mathrm{mL}$ ) and in a previous study $(907 \pm 775 \mathrm{~mL}$ ) [34]. When the study by Yang et al. [20] was omitted from the meta-analysis, the heterogeneity was not significant $\left(P=0.111, I^{2}=44.2 \%\right)$. Influence analysis indicated that the exclusion of this study would not cause the effect size to be overestimated or underestimated (Additional file 4).

When the fixed-effects model was used, the weight of the study by Miyanji et al. [31] was too large (81.82\%), while the sample size was relatively small (23 MISS vs. 23 PSF), which may cause the effect size to be underestimated. To put greater weight on the studies with larger sample sizes, a random-effects model was used.

The remaining six studies included 305 patients in the MISS group and 413 patients in the PSF group. The mean EBL was $288.25 \mathrm{~mL}$ ( $95 \%$ CI 204.96 to 371.54 ) in the MISS group and $517.19 \mathrm{~mL}$ (95\% CI 456.44 to 577.94 ) in the PSF group. The pooled results revealed that the EBL in the MISS group was significantly less than that in the PSF group (WMD, -218.76 ; 95\% CI -256.41 to -181.11 , $P<0.001$ ) (Fig. 3A). Regardless of the model that was used, the statistical significance did not change.

\section{Blood transfusion rate}

Four studies reported the blood transfusion rate. There were 278 patients in the MISS group and 386 patients in the PSF group, with no substantial heterogeneity between groups $\left(P=0.274, I^{2}=22.9 \%\right)$. The mean blood transfusion rate was $8.0 \%$ (95\% CI $0.0 \%$ to $27.0 \%)$ in the MISS group and $35.0 \%$ (95\% CI $9.0 \%$ to $66.0 \%$ ) in the PSF group. The pooled results revealed a significantly lower blood transfusion rate in the MISS group than in the PSF group (RR, $0.31 ; 95 \%$ CI 0.20 to $0.48, P<0.001$ ) (Fig. 3B).

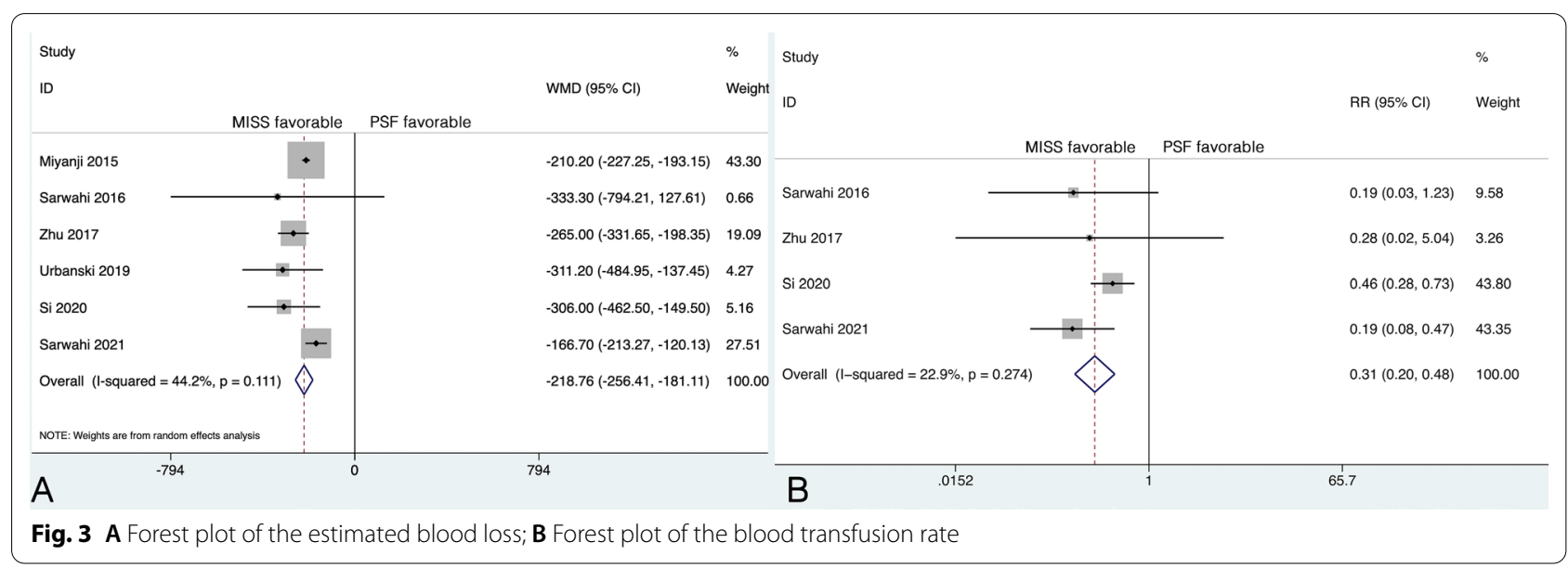




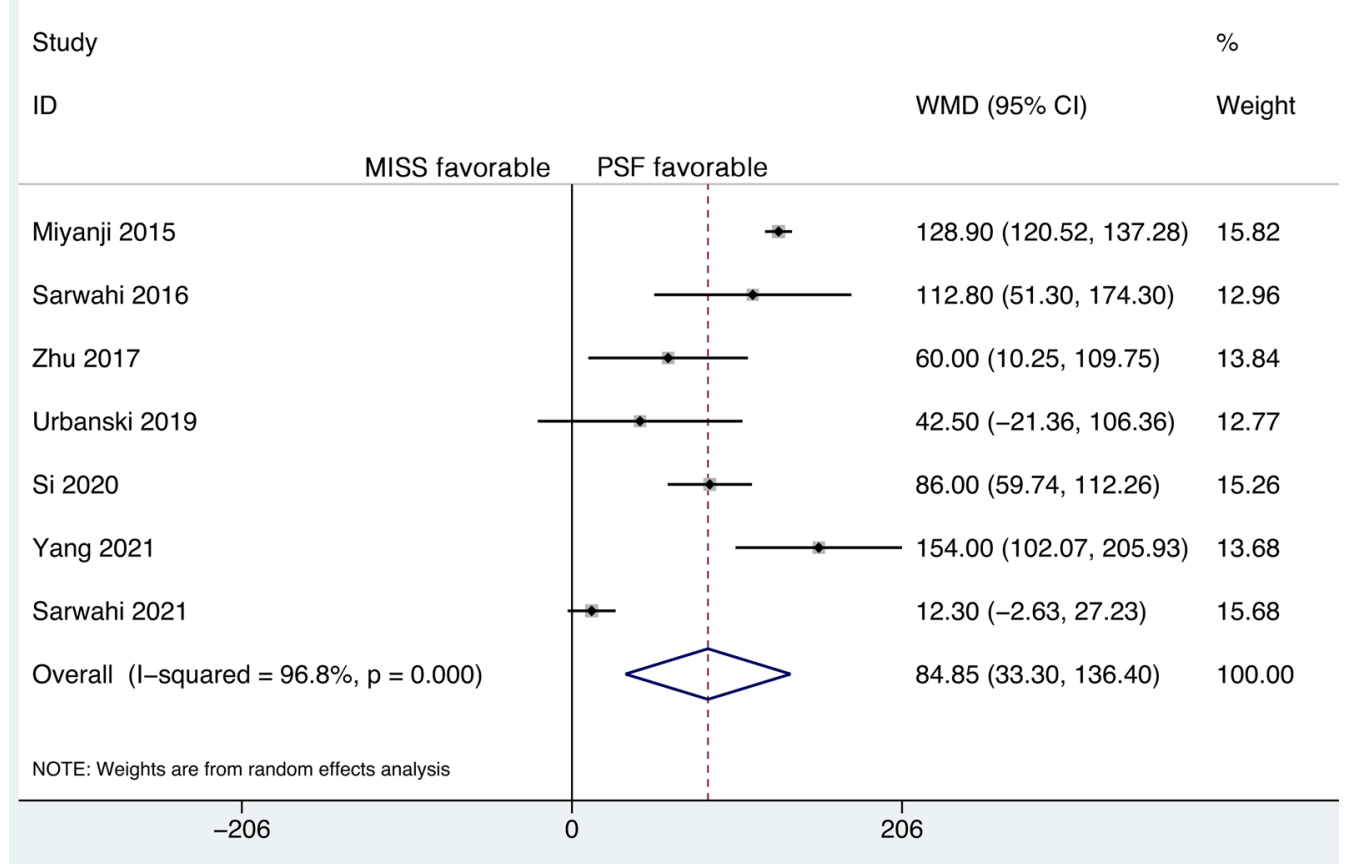

Fig. 4 Forest plot of the operative time

\section{Operative time}

Seven studies reported the ORT, and significant heterogeneity was detected $\left(P<0.001, I^{2}=96.8 \%\right)$. However, further sensitivity analysis did not identify a study that could significantly affect the heterogeneity. Therefore, the random-effects model was applied. The mean ORT was $376.40 \mathrm{~min}$ (95\% CI 293.35 to 459.45 ) in the MISS group and $287.60 \mathrm{~min}$ (95\% CI 237.42 to 337.79 ) in the PSF group. The pooled results revealed a significantly longer ORT in the MISS group than in the PSF group (WMD, 84.85 ; $95 \%$ CI 33.30 to $136.40, P=0.001$ ) (Fig. 4).

\section{Clinical outcomes \\ Length of hospital stay}

Five studies reported the LOS, and significant heterogeneity was detected $\left(P<0.001, I^{2}=94.9 \%\right)$. However, further sensitivity analysis did not identify a study that could significantly affect the heterogeneity. Therefore, the random-effects model was applied.

The five studies included 250 patients in the MISS group and 351 patients in the PSF group. The mean LOS was 6.11 days (95\% CI 4.80 to 7.41 ) in the MISS group and 7.69 days (95\% CI 6.29 to 9.10) in the PSF group. The pooled results revealed a significantly shorter LOS in the MISS group than in the PSF group (WMD, -1.48; 95\% CI -2.48 to $-0.48, P=0.004$ ) (Fig. 5 ).

\section{SRS-22 score}

Four studies reported the overall SRS-22 score after surgery, and significant heterogeneity was detected $\left(P<0.001, I^{2}=90.8 \%\right)$. However, further sensitivity analysis did not identify a study that could significantly affect the heterogeneity. Therefore, the random-effects model was applied. The four studies included 126 patients in the MISS group and 126 patients in the PSF group. The mean overall SRS-22 score was 4.21 (95\% CI 4.18 to 4.25 ) in the MISS group and 4.11 (95\% CI 3.90 to 4.32 ) in the PSF group. The pooled results revealed no significant difference in the overall SRS-22 score between groups (WMD, 0.13 ; $95 \% \mathrm{CI}-0.14$ to $0.41, P=0.339$ ).

Only three of the four studies reported the SRS22 self-image/appearance score and the SRS-22 pain score, including 103 patients in the MISS group and 103 patients in the PSF group. The pooled results revealed no significant difference in the SRS-22 self-image/appearance score between groups (WMD, 0.13; 95\% CI -0.29 to $0.54, P=0.546)$.

\section{Postoperative pain}

Five studies reported postoperative pain. The studies included 146 patients in the MISS group and 168 patients in the PSF group. The SRS-22 pain score was utilized by three studies, and the VAS score was utilized by two studies. Significant heterogeneity was detected $(P=0.001$, 
Study

ID

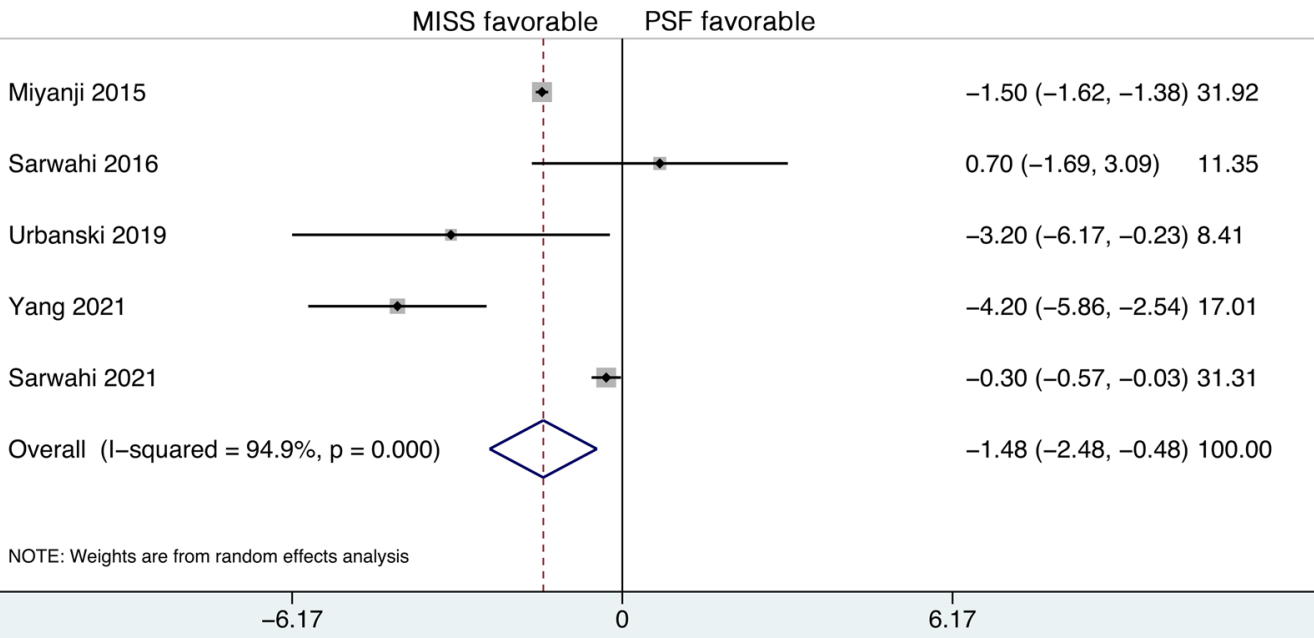

$\%$

WMD $(95 \% \mathrm{Cl}) \quad$ Weight

Fig. 5 Forest plot of the length of hospital stay

Study

ID

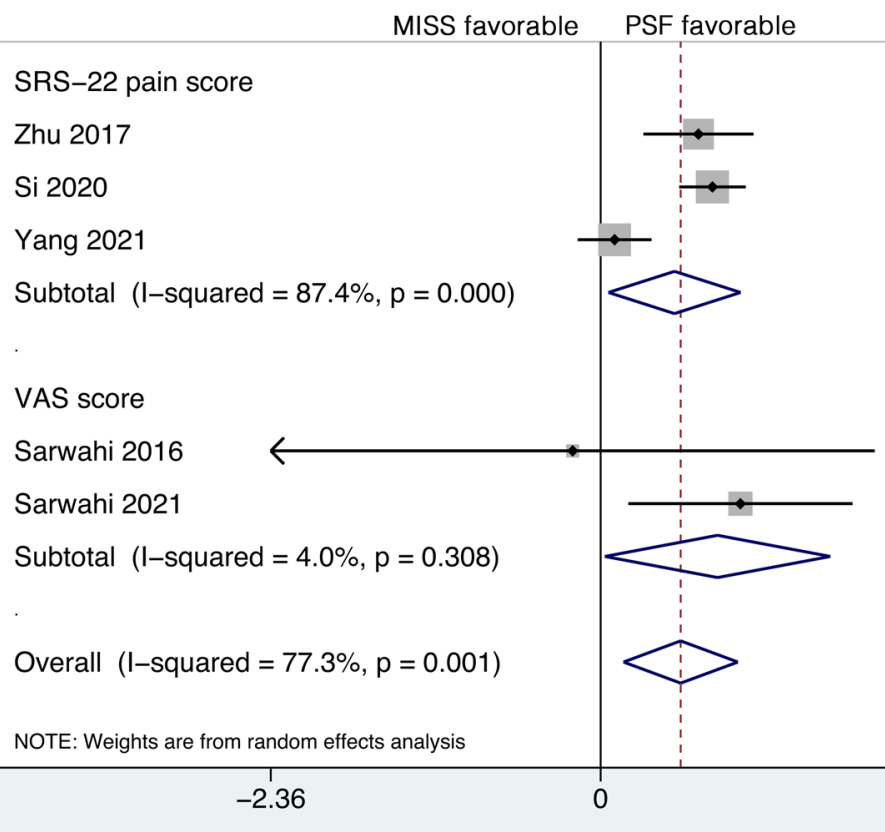

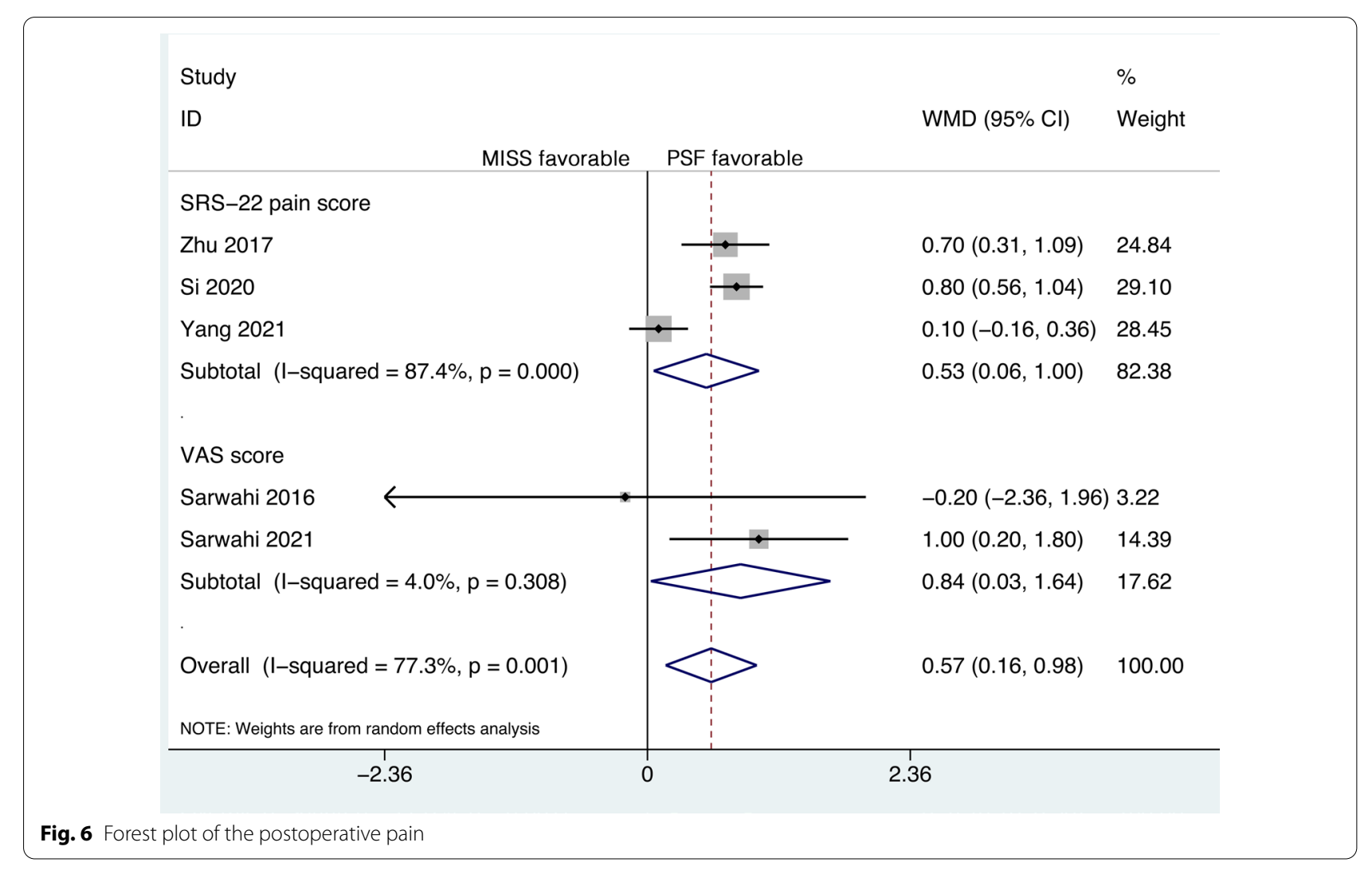


$I^{2}=77.3 \%$ ), and a random-effects model was applied. The pooled results revealed significantly less postoperative pain in the MISS group than in the PSF group (WMD, $0.57 ; 95 \%$ CI 0.16 to $0.98, P=0.006$ ).

To eliminate the influence of different tools, a subgroup analysis was performed. The pooled results revealed significantly less postoperative pain in the MISS group than in the PSF group according to both the SRS-22 pain score (WMD, 0.53 ; $95 \%$ CI 0.06 to $1.00, P=0.028$ ) and the VAS score (WMD, $0.84 ; 95 \%$ CI 0.03 to $1.64, P=0.042$ ) (Fig. 6).

\section{Complications}

\section{Overall complication rate}

Complications, including surgical site infection, hardware failure, wound dehiscence, pseudarthrosis, and hemothorax, were reported by six studies that included 325 patients in the MISS group and 434 patients in the PSF group. No substantial heterogeneity was detected $\left(P=0.496, I^{2}=0.0 \%\right)$. The pooled results revealed no significant difference in the overall complication rate between groups (RR, 1.13 ; $95 \% \mathrm{CI} 0.77$ to $1.67, P=0.521$ ).

\section{Surgical site infection}

Surgical site infection, including superficial and deep wound infection, was reported by six studies with 325 patients in the MISS group and 434 patients in the PSF group. No substantial heterogeneity was detected $\left(P=0.563, I^{2}=0.0 \%\right)$. The pooled results revealed no significant difference in the surgical site infection rate between groups (RR, 1.19; 95\% CI 0.59 to $2.40, P=0.633$ ).

\section{Hardware failure}

Hardware failure, including screw dislodgement, rod dislodgement, and rod breakage, was reported by five studies with 310 patients in the MISS group and 404 patients in the PSF group. No substantial heterogeneity was detected $\left(P=0.679, I^{2}=0.0 \%\right)$. The pooled results revealed no significant difference in the hardware failure rate between groups (RR, 1.12; 95\% CI 0.40 to 3.19, $P=0.828)$.

\section{Subgroup analysis by curve type}

To explore whether the source of heterogeneity was the variety of curve types in AIS patients, we performed a subgroup analysis of the main curve Cobb angle, correction rate, TK, EBL, and ORT according to the curve type (Additional file 5). The heterogeneity of TK and EBL was significantly decreased after the subgroup analysis. The
TK at the last follow-up was significantly greater in the MISS group (WMD, 6.03; 95\% CI 4.46 to $7.60, P<0.001$ ) for patients with mixed curve types (Lenke types 1-6). EBL was significantly lower in the MISS group for Lenke types 1-4 (WMD, -225.22; 95\% CI -293.48 to -156.95 , $P<0.001$ ) and Lenke type 5 patients (WMD, -270.93 ; 95\% CI -333.16 to $-208.70, P<0.001)$. The ORT was significantly longer in the MISS group for Lenke types 1-4 (WMD, 109.33; 95\% CI 67.45 to $151.21, P<0.001$ ) and Lenke type 5 patients (WMD, 53.39; 95\% CI 14.14 to $92.64, P=0.008$ ), while there was no significant difference for patients with mixed curve types (Lenke types $1-6)$.

\section{Subgroup analysis by fusion levels}

To explore whether the source of heterogeneity was the different fusion levels in AIS patients, we performed a subgroup analysis of the main curve Cobb angle, correction rate, TK, EBL, and ORT according to the fusion levels (Additional file 6). The heterogeneity was not significantly decreased after the subgroup analysis. The TK at the last follow-up was significantly greater in the MISS group for patients with $>10$ fusion levels (WMD, 4.24; $95 \%$ CI 1.11 to $7.36, P=0.008$ ). The EBL was significantly lower in the MISS group for patients with $>10$ fusion levels (WMD, -213.09 ; 95\% CI -296.40 to $-129.79, P<0.001$ ) and $\leq 10$ fusion levels (WMD, -275.72; 95\% CI -333.54 to $-217.89, P<0.001)$. The ORT was significantly longer in the MISS group for patients with $>10$ fusion levels (WMD, 100.33; 95\% CI 22.14 to $178.53, P=0.012$ ) and $\leq 10$ fusion levels (WMD, 75.82; 95\% CI 53.86 to 97.79 , $P<0.001)$.

\section{Discussion}

Recently, several studies have demonstrated that AIS could be successfully corrected using minimally invasive techniques [11, 14, 15, 19, 32]. Unlike the percutaneous technique and lateral interbody fusion commonly applied in adult degenerative spinal deformity, MISS for AIS used one to three small skin incisions from a posterior approach, which allowed surgeons to perform facetectomy, instrumentation, and fusion [15, 33]. Compared to standard PSF, dissection of soft tissue and the spinal posterior structure was less common, and the cosmetic concerns of adolescent patients could be relieved [19, 32]. However, as a novel technique, the effectiveness of MISS for the management of AIS still needs to be clarified. In this study, we found that MISS was associated with adequate deformity correction, better restoration of TK, less EBL, lower blood transfusion rate, shorter LOS, and less pain than PSF. 


\section{Correction in the coronal and sagittal planes}

Due to the limited available instrumentation for MISS, the application of compression, distraction, and in situ contouring are still challenges for this technique [31]. However, MISS yielded correction of the main curve Cobb angle, LL, CB, and SVA that was comparable to standard PSF in this meta-analysis. The better restoration of TK in the MISS group, especially for patients with $>10$ fusion levels, was an unexpected finding, although the difference was minor, and whether it had any clinical significance remained debatable. Recently, Sarwahi et al. reported that MISS was associated with significantly greater restoration of TK and a lower postoperative hypokyphosis rate than standard PSF [21]. One plausible explanation was that the preservation of paraspinal muscles and ligaments in the MISS procedure played an important role in maintaining sagittal alignment after surgery [35]. Overall, MISS was adequate to achieve the goal of deformity correction in both the coronal and sagittal planes.

\section{Estimated blood loss}

Minimizing blood loss is of paramount importance for the surgical management of spinal deformity, as the use of allogenic blood transfusion is a risk factor for the development of surgical site infection after spinal fusion [36]. The impact of bleeding-related and transfusionrelated morbidity is even more pronounced in pediatric and adolescent populations. The smaller body size of these patients could accelerate the proportional loss of blood volume during deformity correction surgery for AIS [37]. In a cohort of 188 patients with AIS, Ialenti et al. reported a mean EBL of $907 \pm 775 \mathrm{~mL}$ with standard PSF [34]. In another large cohort of 43,983 patients who underwent PSF for AIS, Yoshihara and Yoneoka reported a blood transfusion rate of $30.4 \%$ [38]. Chiu et al. showed that screw insertion and derotation maneuvers were the main contributors to EBL during standard PSF [39]. These two steps were not modified during the MISS procedure; nonetheless, the EBL and the corresponding need for blood transfusion were significantly decreased in the current study, with an EBL of $288.25 \mathrm{~mL}$ and a blood transfusion rate of $8.0 \%$. We believe that the decreased EBL in the MISS group was attributable to the smaller incisions, reduced tissue dissection, and smaller area of subperiosteal exposure [14]. The ORT was significantly longer in the MISS group than in the PSF group in this study, which reflected the learning curve when applying the new technique [32]. As the longer ORT could also contribute to the increased EBL, we considered that the EBL in the MISS group might continue to decrease with increasing surgeon experience [34, 40].

\section{Length of hospital stay, postoperative pain, and SRS-22} score

This meta-analysis found that the LOS was shorter by 1.48 days in the MISS group than in the PSF group. We considered that this positive outcome was related to the reduced dissection of muscle and EBL in the MISS procedure, which enhanced patient recovery and allowed early discharge. Sultan et al. reported that intraoperative blood loss was an independent risk factor for prolonged LOS in AIS patients undergoing PSF because patients with increased EBL were more likely to develop postoperative complications and require additional management [41]. The shorter LOS may also be tied to patients' postoperative pain and satisfaction $[42,43]$. Martin et al. reported that achieving adequate pain control could decrease the opioid requirement and minimize opioid-induced side effects, allowing AIS patients to mobilize early [44]. Additionally, patients with higher satisfaction tend to return home faster [44]. Consistent with previous studies, we found that patients in the MISS group reported significantly less pain, according to the SRS-22 pain score (4.31 vs. 3.80 ) and the VAS score (5.94 vs. 6.28 ), which was attributed to the reduced dissection of tissue with this new technique. Although the results were not statistically significant, the SRS-22 self-image/appearance score was also higher in the MISS group than in the PSF group (4.05 vs. 3.95). Better pain management and scar appearance could improve overall patient satisfaction, which was reflected by the higher overall SRS-22 score in the MISS group (4.22 vs. 4.11) and led to the shorter LOS in this study.

\section{Complications}

The overall complication profile is an essential aspect of the safety evaluation of a novel surgical technique, particularly in the early stage of application. In this study, the MISS yielded a complication spectrum and complication rate similar to those of standard PSF (15.0\% vs. $18.0 \%)$, suggesting that this new procedure would not cause additional harm and was a safe alternative to conventional open surgery. The most commonly reported complication in both groups was surgical site infection. In this study, the surgical site infection rate was $2.0 \%$ in the MISS group, similar to the PSF group. In a previous study of 540 AIS patients who underwent standard PSF, $2.8 \%$ of cases developed postoperative surgical site infection, which was consistent with our results [45]. Additionally, surgical site infection is associated with ORT [14]. We believe that the decreased ORT of the MISS procedure with increasing surgeon experience would lead to a decrease in the surgical site infection rate. 


\section{Concerns regarding the MISS procedure}

Despite MISS's advantages of better restoration of TK, less EBL, fewer transfusions, shorter LOS, and less postoperative pain, several important concerns must be acknowledged. The ORT was significantly longer with MISS than with PSF (376.40 min vs. $287.60 \mathrm{~min}$ ). The difference in ORT may be the effect of the steep learning curve, and de Bodman et al. and Zhu et al. reported that the ORT of the MISS procedure could be shortened by nearly 60 min with increased surgeon experience $[32,40]$. Nonetheless, even when MISS is performed by a proficient surgeon, the ORT might still be slightly longer than that of standard PSF, and this should be emphasized as a potential limitation of MISS for AIS [33]. Prolonged surgical times keep patients under extended anesthesia, which may have deleterious effects on perioperative morbidity [18].

Concerns have been raised that more fluoroscopic imaging may be needed during the MISS procedure, which could increase the radiation exposure of both patients and surgeons [32, 33]. However, Si et al. reported that a satisfactory screw placement rate of 93.6\% could be obtained using the freehand technique, and no significant difference in radiation exposure was found between the MISS group and the PSF group [19]. Additionally, Zhu et al. achieved a satisfactory screw placement rate of $93.8 \%$ in the MISS group through $\mathrm{O}$-arm navigation, and patients and surgeons could be exposed to less radiation from an $\mathrm{O}$-arm than with traditional fluoroscopy [32].

Appropriate patient selection is important for a new technique, although little is known about the indications of the MISS procedure. To date, most studies have focused on moderate $\left(<80^{\circ}\right)$ and flexible $(>50 \%)$ AIS $[14,15,19,20,31,32]$. Whether the indication can be extended to patients with more severe and rigid curves remains unclear.

\section{Limitations}

This study had several limitations. First, the heterogeneity of the included patients should be acknowledged, as they had different curve types and fusion levels. Two studies focused on Lenke types 1-4, two studies focused on Lenke type 5 , the other three studies included mixed curve types (Lenke type 1-6), and the fusion levels in these studies ranged from 5 to 12 . Heterogeneity may have impacted meaningful pooling. Although we performed subgroup analysis, heterogeneity was still present. Other sources of heterogeneity may have been differences in surgeons, management protocols of spinal centers, and study designs. Second, it is important to address the inherent potential for bias underlying the use of retrospective studies of MISS versus PSF. It is clear that in the absence of randomization, patient selection for MISS or PSF is likely to be a key source of bias in all of the included studies. The patients who are most appropriate for MISS are likely to be selected to undergo MISS, and vice versa. Third, because MISS is a new technique, the results reported by the included studies reflected the early experience of surgeons. Regarding the learning curve of the MISS, de Bodman et al. indicated that the outcomes could be improved after the first 25 cases [40]. However, five of the included studies reported data for their first 25 cases [15, 20, 31-33]. Therefore, the results of this study may be limited when surgeons gain more experience.

\section{Conclusions}

Despite inherent technical challenges, MISS is a feasible and effective alternative to standard PSF for AIS patients with moderate and flexible curves. MISS was associated with adequate deformity correction, better restoration of sagittal alignment, less EBL, fewer transfusions, shorter LOS, and better pain management than PSF. Further research is required to determine the detailed indications for the MISS procedure.

\section{Abbreviations \\ AIS: Adolescent idiopathic scoliosis; PSF: Posterior spinal fusion; MIS: Minimally invasive surgery; MISS: Minimally invasive scoliosis surgery; EBL: Estimated blood loss; ORT: Operative time; LOS: Length of hospital stay; TK: Thoracic kyphosis; LL: Lumbar lordosis; CB: Coronal balance; SVA: Sagittal vertical axis; VAS: Visual analog scale; SRS-22: Scoliosis Research Society-22;WMD: Weighted mean difference; RR: Risk ratio; PRISMA: Preferred Reporting Items for System- atic Reviews and Meta-Analyses.}

\section{Supplementary Information}

The online version contains supplementary material available at https://doi. org/10.1186/s13018-022-02954-4.

Additional file 1. Galbraith plot of the correction rate.

Additional file 2 . Influence analysis of the correction rate.

Additional file 3. Galbraith plot of the estimated blood loss

Additional file 4. Influence analysis of the estimated blood loss.

Additional file 5. Subgroup analysis by curve type.

Additional file 6. Subgroup analysis by fusion levels

Additional file 7. PRISMA 2009 Checklist.

Acknowledgements

We thank Dr. Ziyang Liu for guide in data analysis.

Authors' contributions

$\mathrm{HY}$ and $\mathrm{XJ}$ together conceptualized and designed this study and interpreted the subsequent results, $\mathrm{HY}$ acquired and analyzed the data and drafted the manuscript, $\mathrm{YH}$ gave comments and reviewed the intellectual content, and all authors read and approved the final manuscript. 


\section{Funding}

This research did not receive any specific grants from funding agencies in the public, commercial, or not-for-profit sectors.

\section{Availability of data and materials}

The datasets used and analyzed in the current study can be obtained from the corresponding author on reasonable request.

\section{Declarations}

\section{Ethics approval and consent to participate}

Not applicable.

\section{Consent for publication}

Not applicable.

\section{Competing interests}

The authors declare that they have no competing interests.

Received: 31 August 2021 Accepted: 21 January 2022

Published online: 29 January 2022

\section{References}

1. Weinstein SL. the natural history of adolescent idiopathic scoliosis. J Pediatr Orthop. 2019;39:S44-6. https://doi.org/10.1097/bpo.0000000000 001350.

2. Yu WS, Chan KY, Yu FW, Yeung HY, Ng BK, Lee KM, et al. Abnormal bone quality versus low bone mineral density in adolescent idiopathic scoliosis: a case-control study with in vivo high-resolution peripheral quantitative computed tomography. Spine J. 2013;13:1493-9. https://doi.org/10. 1016/j.spinee.2013.05.018

3. Tambe AD, Panikkar SJ, Millner PA, Tsirikos Al. Current concepts in the surgical management of adolescent idiopathic scoliosis. Bone Joint J. 2018;100-b:415-24. https://doi.org/10.1302/0301-620x.100b4.Bjj-20170846.R2

4. Lonner BS, Ren Y, Yaszay B, Cahill PJ, Shah SA, Betz RR, et al. Evolution of surgery for adolescent idiopathic scoliosis over 20 years: have outcomes improved? Spine (Phila Pa 1976). 2018;43:402-10. https://doi.org/10. 1097/brs.0000000000002332

5. Lonner BS, Kondrachov D, Siddiqi F, Hayes V, Scharf C. Thoracoscopic spinal fusion compared with posterior spinal fusion for the treatment of thoracic adolescent idiopathic scoliosis. J Bone Joint Surg Am. 2006;88:1022-34. https://doi.org/10.2106/jbjs.E.00001.

6. Lonner BS, Brochin R, Lewis R, Vig KS, Kassin G, Castillo A, et al. Body image disturbance improvement after operative correction of adolescent idiopathic scoliosis. Spine Deform. 2019;7:741-5. https://doi.org/10. 1016/j.jspd.2018.12.005.

7. Virk S, Wright-Chisem J, Sandhu M, Vaishnav A, Albert TJ, Gang CH, et al. A novel magnetic resonance imaging-based lumbar muscle grade to predict health-related quality of life scores among patients requiring surgery. Spine (Phila Pa 1976). 2021;46:259-67. https://doi.org/10.1097/brs.00000 00000003833

8. Patel PD, Canseco JA, Houlihan N, Gabay A, Grasso G, Vaccaro AR. Overview of minimally invasive spine surgery. World Neurosurg. 2020;142:4356. https://doi.org/10.1016/j.wneu.2020.06.043.

9. Phan K, Mobbs RJ. Minimally invasive versus open laminectomy for lumbar stenosis: a systematic review and meta-analysis. Spine (Phila Pa 1976). 2016;41:E91-e100. https://doi.org/10.1097/brs.0000000000001161

10. Dangelmajer S, Zadnik PL, Rodriguez ST, Gokaslan ZL, Sciubba DM. Minimally invasive spine surgery for adult degenerative lumbar scoliosis. Neurosurg Focus. 2014;36:E7. https://doi.org/10.3171/2014.3.Focus144.

11. Sarwahi V, Wollowick AL, Sugarman EP, Horn JJ, Gambassi M, Amaral TD. Minimally invasive scoliosis surgery: an innovative technique in patients with adolescent idiopathic scoliosis. Scoliosis. 2011;6:16. https://doi.org/ 10.1186/1748-7161-6-16.

12. Barbanti Brodano G, Martikos K, Vommaro F, Greggi T, Boriani S. Less invasive surgery in idiopathic scoliosis: a case report. Eur Rev Med Pharmacol Sci. 2014;18:24-8.
13. Gómez H, Burgos J, Hevia E, Maruenda JI, Sanpera I. Immediate postoperative and long-term results of a minimally invasive approach for the correction of adolescent idiopathic scoliosis. Coluna/ Columna. 2012;12:291-5. https://doi.org/10.1590/S1808-18512013000400005.

14. de Bodman C, Miyanji F, Borner B, Zambelli PY, Racloz G, Dayer R. Minimally invasive surgery for adolescent idiopathic scoliosis: correction of deformity and peri-operative morbidity in 70 consecutive patients. Bone Joint J. 2017;99-b:1651-7. https://doi.org/10.1302/0301-620x.99b12.Bjj2017-0022.R2

15. Sarwahi V, Horn JJ, Kulkarni PM, Wollowick AL, Lo Y, Gambassi M, et al. Minimally invasive surgery in patients with adolescent idiopathic scoliosis: is it better than the standard approach? a 2-year follow-up study. Clin Spine Surg. 2016;29:331-40. https://doi.org/10.1097/bsd.0000000000 000106.

16. Harrison Farber S, Nayar G, Desai R, Reiser EW, Byrd SA, Chi D, et al. Radiation exposure to the surgeon during minimally invasive spine procedures is directly estimated by patient dose. Eur Spine J. 2018;27:1911-7. https:// doi.org/10.1007/s00586-018-5653-6.

17. Tan JH, Wong HK. Minimally invasive options in adolescent idiopathic scoliosis. Indian Spine J. 2020;3:207. https://doi.org/10.4103/isj.isj_63_19.

18. Alhammoud A, Alborno Y, Baco AM, Othman YA, Ogura Y, Steinhaus M, et al. Minimally invasive scoliosis surgery is a feasible option for management of idiopathic scoliosis and has equivalent outcomes to open surgery: a meta-analysis. Global Spine J. 2021:2192568220988267. https:// doi.org/10.1177/2192568220988267

19. Si G, Li T, Wang Y, Liu X, Li C, Yu M. Minimally invasive surgery versus standard posterior approach for Lenke Type 1-4 adolescent idiopathic scoliosis: a multicenter, retrospective study. Eur Spine J. 2021;30:706-13. https://doi.org/10.1007/s00586-020-06546-w.

20. Yang JH, Kim HJ, Chang DG, Suh SW. Comparative analysis of radiological and clinical outcomes between conventional open and minimally invasive scoliosis surgery for adolescent idiopathic scoliosis. World Neurosurg. 2021. https://doi.org/10.1016/j.wneu.2021.04.025.

21. Sarwahi V, Galina JM, Hasan S, Atlas A, Ansorge A, De Bodman C, et al. Minimally invasive versus standard surgery in idiopathic scoliosis patients: a comparative study. Spine (Phila Pa 1976). 2021;46:1326-35. https://doi. org/10.1097/brs.0000000000004011

22. Moher D, Liberati A, Tetzlaff J, Altman DG. Preferred reporting items for systematic reviews and meta-analyses: the PRISMA statement. BMJ. 2009;339: b2535. https://doi.org/10.1136/bmj.b2535.

23. Stang A. Critical evaluation of the Newcastle-Ottawa scale for the assessment of the quality of nonrandomized studies in meta-analyses. Eur J Epidemiol. 2010;25:603-5. https://doi.org/10.1007/s10654-010-9491-z.

24. Lenke LG, Betz RR, Harms J, Bridwell KH, Clements DH, Lowe TG, et al. Adolescent idiopathic scoliosis: a new classification to determine extent of spinal arthrodesis. J Bone Joint Surg Am. 2001;83:1169-81. https://doi. org/10.1590/S0104-14281998000400006.

25. Asher M, Min Lai S, Burton D, Manna B. The reliability and concurrent validity of the scoliosis research society-22 patient questionnaire for idiopathic scoliosis. Spine (Phila Pa 1976). 2003;28:63-9. https://doi.org/10. 1097/00007632-200301010-00015

26. Breivik H, Borchgrevink PC, Allen SM, Rosseland LA, Romundstad L, Hals EK, et al. Assessment of pain. Br J Anaesth. 2008;101:17-24. https://doi. org/10.1093/bja/aen103.

27. Higgins JP, Thompson SG, Deeks JJ, Altman DG. Measuring inconsistency in meta-analyses. Bmj. 2003;327:557-60. https://doi.org/10.1136/bmj.327. 7414.557.

28. Li B, Li F, Wang L, Zhang D. Fruit and vegetables consumption and risk of hypertension: a meta-analysis. J Clin Hypertens (Greenwich). 2016;18:468-76. https://doi.org/10.1111/jch.12777.

29. Egger M, Davey Smith G, Schneider M, Minder C. Bias in meta-analysis detected by a simple, graphical test. BMJ. 1997;315:629-34. https://doi. org/10.1136/bmj.315.7109.629.

30. Duval S, Tweedie R. Trim and fill: A simple funnel-plot-based method of testing and adjusting for publication bias in meta-analysis. Biometrics. 2000;56:455-63. https://doi.org/10.1111/j.0006-341x.2000.00455.x.

31. Miyanji F, Desai S. Minimally invasive surgical options for adolescent idiopathic scoliosis. Seminars Spine Surgery. 2015;27:39-44. https://doi.org/ 10.1053/j.semss.2015.01.009.

32. Zhu W, Sun W, Xu L, Sun X, Liu Z, Qiu Y, et al. Minimally invasive scoliosis surgery assisted by $\mathrm{O}$-arm navigation for Lenke Type 5C adolescent 
idiopathic scoliosis: a comparison with standard open approach spinal instrumentation. J Neurosurg Pediatr. 2017;19:1-7. https://doi.org/10. 3171/2016.11.PEDS16412.

33. Urbanski W, Zaluski R, Kokaveshi A, Aldobasic S, Miekisiak G, Morasiewicz P. Minimal invasive posterior correction of Lenke 5C idiopathic scoliosis: comparative analysis of minimal invasive vs. open surgery. Arch Orthop Trauma Surg. 2019;139:1203-8. https://doi.org/10.1007/ s00402-019-03166-y

34. Ialenti MN, Lonner BS, Verma K, Dean L, Valdevit A, Errico T. Predicting operative blood loss during spinal fusion for adolescent idiopathic scoliosis. J Pediatr Orthop. 2013;33:372-6. https://doi.org/10.1097/BPO.0b013 e3182870325.

35. Kuo YK, Lin YC, Lee CY, Chen CY, Tani J, Huang TJ, et al. Novel Insights into the Pathogenesis of spinal sarcopenia and related therapeutic approaches: a narrative review. Int J Mol Sci. 2020;21. https://doi.org/10. 3390/ijms21083010

36. Woods Bl, Rosario BL, Chen A, Waters JH, Donaldson W 3rd, Kang J, et al. The association between perioperative allogeneic transfusion volume and postoperative infection in patients following lumbar spine surgery. J Bone Joint Surg Am. 2013;95:2105-10. https://doi.org/10.2106/jbjs.L. 00979.

37. Jain A, Sponseller PD, Newton PO, Shah SA, Cahill PJ, Njoku DB, et al. Smaller body size increases the percentage of blood volume lost during posterior spinal arthrodesis. J Bone Joint Surg Am. 2015;97:507-11. https://doi.org/10.2106/jbjs.N.01104.

38. Yoshihara $\mathrm{H}$, Yoneoka D. National trends in spinal fusion for pediatric patients with idiopathic scoliosis: demographics, blood transfusions, and in-hospital outcomes. Spine (Phila Pa 1976). 2014;39:1144-50. https://doi. org/10.1097/brs.0000000000000354

39. Chiu CK, Chan CY, Aziz I, Hasan MS, Kwan MK. Assessment of intraoperative blood loss at different surgical stages during posterior spinal fusion surgery in the treatment of adolescent idiopathic scoliosis. Spine (Phila Pa 1976). 2016;41:E566-73. https://doi.org/10.1097/brs.00000000000001304

40. de Bodman C, Ansorge A, Tabard A, Amirghasemi N, Dayer R. Clinical and radiological outcomes of minimally-invasive surgery for adolescent idiopathic scoliosis at a minimum two years' follow-up. Bone Joint J. 2020;102-b:506-12. https://doi.org/10.1302/0301-620x.102b4.Bjj-20190447.R1

41. Sultan AA, Berger RJ, Cantrell WA, Samuel LT, Ramanathan D, Churchill $J$, et al. Predictors of extended length of hospital stay in adolescent idiopathic scoliosis patients undergoing posterior segmental instrumented fusion: an analysis of 407 surgeries performed at a large academic center. Spine (Phila Pa 1976). 2019;44:715-22. https://doi.org/10.1097/brs.00000 00000002919

42. Sanders AE, Andras LM, Sousa T, Kissinger C, Cucchiaro G, Skaggs DL. Accelerated discharge protocol for posterior spinal fusion patients with adolescent idiopathic scoliosis decreases hospital postoperative charges 22. Spine (Phila Pa 1976). 2017;42:92-7. https://doi.org/10.1097/brs.00000 00000001666

43. Tsai TC, Orav EJ, Jha AK. Patient satisfaction and quality of surgical care in US hospitals. Ann Surg. 2015;261:2-8. https://doi.org/10.1097/sla.00000 00000000765

44. Martin BD, Pestieau SR, Cronin J, Gordish-Dressman H, Thomson K, Oetgen ME. Factors affecting length of stay after posterior spinal fusion for adolescent idiopathic scoliosis. Spine Deform. 2020;8:51-6. https://doi. org/10.1007/s43390-020-00042-3.

45. Di Silvestre M, Bakaloudis G, Lolli F, Giacomini S. Late-developing infection following posterior fusion for adolescent idiopathic scoliosis. Eur Spine J. 2011;20(Suppl 1):S121-7. https://doi.org/10.1007/s00586-011-1754-1.

\section{Publisher's Note}

Springer Nature remains neutral with regard to jurisdictional claims in published maps and institutional affiliations. 